\title{
La inserción de las mujeres inmigradas en el mercado de trabajo español
}

\author{
Elena Vidal \\ Universitat Pompeu Fabra. Departament de Ciències Polítiques i Socials \\ elena.vidalcoso@upf.edu \\ Daniela Vono \\ Centre d'Estudis Demogràfics \\ dvono@ced.uab.es
}

Recepción: octubre de 2010

Aceptación: octubre de 2010

\section{Resumen}

La creciente presencia femenina entre la población inmigrada en España ha hecho necesario replantearse el estudio de la misma desde una perspectiva de género. En el presente trabajo, se hace un recorrido por las principales aportaciones científicas centradas en la inmigración femenina y, de manera específica, en su inserción en el mercado de trabajo español. Se identifica la demanda creciente de trabajadoras para el servicio doméstico como la razón principal de la llegada de las mujeres no comunitarias, dentro de la internacionalización de las funciones de reproducción social. El resultado ha sido la existencia de la complementariedad sociodemográfica entre la mano de obra femenina inmigrada y la autóctona, dadas las diferencias en sus patrones de actividad y en las posiciones laborales que ocupan. Las siguientes páginas reúnen, asimismo, los principales estudios sobre la movilidad laboral, desde el servicio doméstico hacia otras actividades económicas de las trabajadoras inmigradas.

Palabras clave: inmigración femenina; trabajo; servicio doméstico; segmentación; complementariedad.

Resum. La inserció de les dones immigrades en el mercat de treball espanyol

L'augment de la presència femenina entre la població immigrada a Espanya ha fet necessari replantejar-se'n l'estudi des de la perspectiva de gènere. En aquest treball, s'hi realitza un recorregut per les principals aportacions científiques centrades en la immigració femenina i, de manera més concreta, en la seva inserció en el mercat de treball espanyol. S’hi identifica la creixent demanda de treballadores per al servei domèstic com la raó principal de l'arribada de les dones no comunitàries, en el context de la internacionalització de les funcions de reproducció social. Ateses les diferències en els patrons d'activitat i en les posicions laborals que ocupen, existeix una complementarietat sociodemogràfica entre la mà d'obra femenina immigrada i l'autòctona. Les pàgines següents reuneixen, així mateix, els estudis principals sobre la mobilitat laboral de les treballadores immigrades, des del servei domèstic fins a unes altres activitats econòmiques.

Paraules clau: immigració femenina; treball; servei domèstic; segmentació; complementarietat. 
Résumé. L'insertion sur le marché du travail espagnol des femmes immigrantes

L'introduction de la perspective de genre dans les études sur la migration en Espagne a été nécessaire du fait de l'intensification de l'immigration féminine. Cet article présente les principales contributions scientifiques à l'étude des migrations des femmes, et plus spécifiquement, à l'étude de l'insertion de cette population sur le marché du travail espagnol. La plupart des auteurs ont identifié le besoin en main-d'œuvre destinées aux tâches ménagères comme la principale cause de cette immigration de femmes non-communautaires, dans le cadre d'une internalisation du processus de reproduction sociale. Il existe une complémentarité entre les forces de travail féminines natives et immigrées compte tenu des différences de parcours professionnels et de postes occupées. Les pages qui suivent résument l'étude la plus importante sur la mobilité des femmes sur le marché du travail, des tâches ménagères vers d'autres activités.

Mots clé: migration des femmes; marché du travail; tâches ménagères; segmentation; complémentarité.

\section{Abstract. Labour insertion of immigrant women in the Spanish Labour Market}

The increasing number of female immigrants in Spain made the introduction of the gender perspective in its analysis necessary. This paper quotes the main scientific contributions to the study of immigrant women and, more specifically, in their insertion in the Spanish Labour Market. Demand of female workers for the domestic service, is identified by most of the authors, as the most important cause of arrival of non-communitarian women. Therefore, it would be part of the internationalization of the social reproduction process. Differences between activity trends and labour positions indicate that there is socio-demographic complementarity between the immigrant and the native female labour force The next pages summarize the most notorious study on immigrant women's labour mobility from domestic service to other activities.

Key words: female immigration; labour; domestic service; segmentation; complementarity.

\section{Sumario}

La inmigración femenina como respuesta a una demanda específica de mano de obra

La complementariedad con las trabajadoras autóctonas
La participación laboral de las mujeres inmigradas

¿Trabajadoras domésticas por siempre jamás? La movilidad laboral

Referencias bibliográficas

\section{La inmigración femenina como respuesta a una demanda específica de mano de obra}

A día de hoy, es impensable referirse a la población inmigrada como a una población masculina. La creciente presencia femenina ha hecho necesario replantearse el estudio de la misma desde una perspectiva de género. Tal orientación se hace imprescindible, no solamente por el volumen poblacional que esas mujeres representan, sino también para comprender tanto las motivaciones que las han llevado a escoger España como destino migratorio, como 
las consecuencias socioeconómicas que se derivan de su inserción en nuestro mercado de trabajo. Solamente una década atrás, Domingo (1998) nos hacía reflexionar sobre la imagen que para aquel entonces teníamos de la inmigración extranjera en España: «Un hombre, joven y posiblemente magrebí» (Domingo, 1998: 11). La invisibilidad de las mujeres inmigradas en el imaginario colectivo contrastaba con la creciente visibilidad social de las mismas. En consecuencia, Domingo y Brancós (1999) pronosticaron un futuro en que la presencia de la población femenina de nacionalidad extranjera sería más perceptible, tanto social como estadísticamente, efecto del proceso de globalización y de las tendencias flexibilizadoras del mercado de trabajo español.

Y, efectivamente, en los años más recientes, se ha acrecentado notablemente la producción científica centrada en la inmigración femenina y, de manera específica, en su inserción en el mercado de trabajo español. Con la llegada del nuevo milenio, son muchos los autores que identifican las especificidades de la demanda laboral en España como la razón principal de la feminización de los flujos migratorios, con lo cual se alejan de la explicación de los mismos como respuesta a los procesos de reagrupación familiar. Gaspard (2000), por ejemplo, identifica la inmigración femenina como un fenómeno vinculado a la importancia del sector servicios en los países de destino. En la misma línea, Juliano (2000) afirma que la selección por género de los flujos inmigratorios es una clara respuesta a una demanda de mano de obra específica. Izquierdo (2000) sostiene que los proyectos migratorios de hombres y mujeres en España no son tan divergentes en función del sexo, ya que el motivo principal de ambos es laboral, lejos del estereotipo según el cual para los hombres es el trabajo y para las mujeres, la familia.

Otros autores (Cachón, 1997; Parella, 2002) van un paso más lejos y aseguran que es el mismo Estado el que actúa, a través de su política migratoria, de canalizador de los flujos de mujeres inmigrantes procedentes de los países no comunitarios. La política de contingentes ha seleccionado a los inmigrantes tanto en función de su nacionalidad, como de su sexo, según sea la actividad económica en la que van a trabajar. Para estos autores, la política migratoria de España ha ido dirigida a promover la inmigración femenina laboral, lo cual responde a la demanda existente de trabajadoras para el servicio doméstico. Por tanto, para los autores, el factor más sustancial para entender porque inmigran las mujeres a España es la naturaleza del mercado de trabajo de acogida.

De acuerdo con las explicaciones del mercado de trabajo dual o segmentado de Piore (1971, 1975, 1979a y 1979b), Martínez Veiga (1997) indica que las especificidades de la inserción laboral de las mujeres inmigrantes se explicarían por la multiplicidad de ejes de segmentación en el mercado de trabajo español. Siguiendo la misma línea argumental, diversos autores añaden la variable sexo, que, junto al origen migratorio o nacional, van a constituir los dos elementos o ejes clave a partir de los que se explican las posiciones laborales ocupadas por las mujeres inmigradas en España (Oso, 1998; Catarino y Oso, 2000; Juliano, 2000; Colectivo Ioé, 2001a; Solé 2001 y 2003; Parella, 2002). Estos autores sostienen que, si bien el colectivo de trabajadores inmigrados se encuentra 
en una situación de clara subordinación, puesto que se insertan en aquellas posiciones laborales que son rechazadas por la población autóctona, las que son propias del mercado de trabajo secundario, esta segmentación se produce siguiendo una especificación de género. Mientras los varones inmigrados se ocupan en esas vacantes laborales que no interesan a los hombres autóctonos, las mujeres inmigradas se emplean en las que no interesan a las mujeres autóctonas. Para estos autores, el género incorpora otra dimensión en la estratificación de la fuerza de trabajo: además de ser inmigradas, estas mujeres experimentan dificultades adicionales por el hecho de ser mujer. Por tanto, es lógico pensar que parte de las tareas reproductivas que tradicionalmente estaban asignadas a la población femenina autóctona son ocupadas actualmente por las mujeres inmigradas, con lo cual se produce una división étnica de esas actividades. Según los autores antes citados, la demanda de trabajo femenino inmigrante responde, en la mayoría de los casos, a la internacionalización de las funciones de reproducción social, de la que España se ha convertido en un caso paradigmático durante los últimos años. De ese modo, no resulta extraño que el sector de actividad económica por excelencia de inserción laboral de las inmigradas sea el servicio doméstico.

La explicación de la fuerte demanda en España de trabajadoras inmigrantes para el servicio doméstico estriba en el envejecimiento de la población, en el cambio de las estructuras familiares, en la transformación del rol social de la mujer autóctona, así como en la naturaleza deficiente del Estado de bienestar de los países del sur de Europa (Kofman, 1999; Arango, 2004; Fernández Cordón, 2004). Tal como afirma Parella (2002), dado que, en España, las trabajadoras autóctonas dedicadas al servicio doméstico exigen salarios más elevados que las extranjeras, y muchas familias no pueden o no quieren pagar precios tan elevados, las trabajadoras de origen inmigrante constituyen la alternativa más rentable. A parte de su competitividad en precios, otro factor que explica la creciente demanda de inmigradas es la escasez de autóctonas dispuestas a trabajar en esos servicios, consecuencia de la extraordinaria mejora de los niveles educativos de las generaciones más jóvenes.

Siguiendo con el análisis de las actividades de inserción más comunes, el Colectivo Ioé (2001b) identificaba la hostelería como la segunda opción laboral de las mujeres extranjeras no comunitarias. Según estos autores, las tareas que estas mujeres tenían asignadas dentro del sector estaban más relacionadas con actividades de limpieza y en la cocina, sin ser tan presentes en trabajos que requerían contacto directo con la clientela, como los de camarera, que eran reservados a las españolas. En cambio, King y Zontini (2000) identificaban el mercado del sexo como el segundo sector de ocupación de las mujeres inmigradas en el sur de Europa, después del servicio doméstico. Sin embargo, los autores indican los problemas para cuantificar la importancia que la prostitución tiene como sector de ocupación de esas mujeres, dada la falta de datos, consecuencia de su naturaleza sensible, por ser una actividad de la economía sumergida, así como por la movilidad geográfica y la alta estacionalidad de la misma. No obstante, contamos con aportaciones como la de Carmona (2000), 
que estudia la prostitución de mujeres inmigradas en el barrio barcelonés del Raval. Una de las conclusiones de la autora es que la decisión de prostituirse es, muchas veces, fruto de una estrategia migratoria basada en ganar cuanto más dinero mejor en una primera fase $\mathrm{y}$, de ese modo, poder hacer realidad otros proyectos a largo plazo.

En el caso particular de las latinoamericanas, que son las mujeres extranjeras que más se han incorporado al mercado de trabajo español, Vono (2010), a partir de los datos de la Encuesta de Población Activa, encuentra que estas se concentran fundamentalmente en el servicio doméstico y en servicios de limpieza (un 40\% del total de ocupadas en 2001 y un 38\% en 2009). En segundo lugar, están las ocupaciones relacionadas con el sector servicios: un 16\% de las latinoamericanas ocupadas en 2009 se dedicaban a servicios de restauración; un $11 \%$, a servicios personales, y un $7 \%$, a trabajos de dependientes de comercio.

\section{La complementariedad con las trabajadoras autóctonas}

El doble proceso discriminatorio al que están sometidas las trabajadoras inmigrantes se produce, no solo respecto al sexo masculino, sino también respecto a las trabajadoras autóctonas, de las cuales se convierten en complementarias. Las inmigradas se ocupan principalmente en las posiciones típicas del mercado de trabajo secundario, mientras las españolas lo hacen en las del mercado primario, más estables, prestigiosas y cualificadas. Anthias (2000) sostiene que las mujeres autóctonas pueden mejorar su posición en el mercado de trabajo y, por tanto, su estatus social, gracias a que son, en muchas ocasiones, las mujeres inmigrantes las que realizan el trabajo reproductivo que ellas rechazan. La inmigración de las mujeres extranjeras en España es consecuencia directa, según Solé (2003), de la elevación del nivel de aceptabilidad laboral de las mujeres autóctonas a partir de los años ochenta, fruto de la mejora del nivel educativo de las más jóvenes. La autora argumenta que las trabajadoras inmigradas van a ocuparse en aquellas posiciones laborales cualificadas, de acuerdo con su nivel educativo, lo cual provoca un déficit entre la demanda y la oferta de trabajadoras poco cualificadas, especialmente en el servicio doméstico. En este contexto, las mujeres inmigradas llegan a nuestro país respondiendo a unas demandas laborales específicas.

Domingo et al. (2007) analizan esta complementariedad a nivel provincial. Las conclusiones de su estudio confirman la existencia de altos índices de mujeres de nacionalidad extracomunitaria precisamente en aquellas provincias con un mayor nivel de instrucción y unas mayores tasas de actividad de las mujeres residentes. Vidal (2009), en su estudio sobre la actividad económica femenina a través de los datos transversales de la Encuesta de Población Activa (EPA), apoya la idea de la existencia de una complementariedad sociodemográfica en función del origen, en que la llegada de mujeres inmigradas ayuda a la promoción de las mujeres autóctonas. La autora sostiene que la coexistencia de las crecientes tasas de actividad de las jóvenes españolas y la alta cualificación de sus ocupaciones, con los también altos índices de participación laboral de 
las inmigradas y su casi exclusiva ocupación en las tareas tradicionalmente reproductivas, apunta a la complementariedad entre las mujeres en el mercado de trabajo según el origen nacional. En los resultados de su estudio, se observa un aumento, durante la última década, de la segmentación de la estructura ocupacional femenina en función del lugar de nacimiento. Así, mientras se observa un aumento considerable de las autóctonas trabajando en las posiciones más cualificadas del mercado laboral, este proceso ha coincidido en el tiempo con el espectacular crecimiento de las entradas de inmigradas ocupadas como empleadas domésticas y en la restauración. Adicionalmente, si la cualificación de las ocupaciones de las autóctonas depende de su nivel educativo, el análisis demuestra que, en el caso de las inmigradas, la probabilidad de trabajar en las posiciones a la base de la escala ocupacional es independiente del nivel formativo de la mujer.

\section{La participación laboral de las mujeres inmigradas}

Si bien es cierto, como apuntan los autores antes citados, que la inmigración femenina en España tiene una finalidad eminentemente económica y laboral, también es cierto que la participación laboral de algunos colectivos de mujeres inmigradas es muy inferior a la mediana. Parella y Samper (2007), teniendo en cuenta la importancia del grado de participación de las mujeres en el trabajo reproductivo, sostienen que las mujeres inmigradas presentan los mismos problemas conciliatorios que las autóctonas. No obstante, las primeras cuentan con menos recursos, tanto familiares como económicos, para hacer frente a estas dificultades. Además, para estas autoras, lo restrictivo de las oportunidades laborales a las que pueden optar, independientemente de su nivel educativo, aumenta el coste de oportunidad de su participación laboral. Por dichos motivos, las autoras no encuentran extraño que algunas inmigradas opten por no trabajar, especialmente cuando tienen responsabilidades familiares.

Por otro lado, Oso (1998) afirma que no se puede entender el grado de participación laboral de los diversos colectivos de mujeres inmigradas, sin tener en cuenta el tipo de flujo migratorio, que, a su vez, es un reflejo de las estructuras patriarcales y las jerarquías de poder intrafamiliares imperantes en los mismos. La línea argumental de la autora es que las estrategias migratorias masculinizadas o feminizadas determinan el grado de participación laboral en la sociedad de acogida. Cuando el hombre es el pionero de la migración familiar y la mujer migra por reagrupamiento familiar, el caso mayoritario entre el colectivo marroquí, por ejemplo, la mujer acabada de llegar es legal y económicamente dependiente de su marido y acostumbra a quedarse en el ámbito doméstico, al menos durante la primera etapa posterior a la migración. En cambio, cuando la migración es conjunta de la pareja, la dependencia respecto al sexo masculino es menor y la inserción en el mercado de trabajo es más pronta. Finalmente, cuando la mujer es la pionera de la cadena migratoria, el caso mayoritario del colectivo latinoamericano, la autonomía social y económica de la mujer es mucho mayor. 
Vidal (2009) estudia las diferencias existentes entre los patrones de participación laboral de las mujeres autóctonas y los de las mujeres inmigradas. Sus resultados apuntan que son las variables familiares y las características de su estrategia migratoria las que explican la menor o mayor propensión al trabajo extradoméstico de las mujeres inmigradas, mientras la edad o la generación y el nivel educativo son cuestiones clave para entender la participación en el mercado laboral de las autóctonas. Entre las mujeres de origen extranjero, hay situaciones muy diversas respecto a su participación laboral. Por un lado, las latinoamericanas y las de la Europa del Este, con unas tasas de actividad altísimas a todas las edades, con independencia del nivel formativo. Por otro lado, las africanas, con una baja participación laboral y muy influenciada por sus características familiares o, lo que es lo mismo, por su convivencia en pareja, así como por la presencia de hijos dependientes en el hogar. El análisis también ha confirmado que, para todos los colectivos, a mayor tiempo residiendo en España, mayor es la propensión al trabajo extradoméstico, así como la importancia del tipo de migración familiar. Efectivamente, ser la pionera de la migración familiar tiene efectos clave para conseguir una mayor autonomía laboral, especialmente en comparación con las reagrupadas o aquellas que forman una pareja mixta con españoles, menos propensas a la participación laboral.

\section{¿Trabajadoras domésticas por siempre jamás? La movilidad laboral}

Tal como hemos indicado, el crecimiento en España de la demanda de trabajadoras inmigrantes para las tareas reproductivas explicaría que el servicio doméstico constituya la principal puerta de entrada de esas mujeres al mercado de trabajo español. Eso conlleva, para la mayoría de las trabajadoras domésticas inmigradas, un proceso de movilidad socioprofesional descendiente respecto a la posición de ocupada en la sociedad de origen. La explicación para el caso concreto español reside, según Oso (2003), en la noción de estrategia social, a través de la cual, y actuando racionalmente, las mujeres inmigradas aceptan trabajar en el servicio doméstico como parte de una etapa inicial del proyecto migratorio. El trabajo doméstico es el primer paso hacia unos objetivos a largo plazo. Esta estrategia explicaría la aceptación, nada más llegar, de una situación precaria y de una movilidad social descendiente respecto a la ocupada en el país de origen. Solé (2003) concuerda con la autora al sostener que la aceptación de esta situación por parte de la mujer extranjera se debe entender como una estrategia para poder regularizar su situación jurídica, percibir unos ingresos, poder ahorrar y enviar remesas económicas a sus familias. Así mismo, apunta a la importancia de la idea que esas mujeres tienen de su migración de retorno, así como de la falta de red de ayuda económica y familiar en la sociedad de destino. Todos estos elementos, a juicio de la autora, les impiden ser más selectivas con los trabajos que escogen, lo cual rebaja su nivel de aceptabilidad laboral.

Oso (2003) realiza una serie de entrevistas a mujeres inmigrantes con el fin de estudiar sus estrategias de movilidad sociolaboral. Los resultados de la investigación apuntan que, para las inmigradas que se insertaron en el merca- 
do de trabajo español a través del servicio doméstico, las pocas alternativas de movilidad ocupacional se limitaban a pasar del servicio doméstico interno al externo, aunque en algunos casos se observa un cambio a la restauración. Otra de sus conclusiones es que aquellas que llevaban menor tiempo residiendo en España, las endeudadas, las irregulares y las que tenían mayor necesidad de enviar remesas, eran las se encontraban en una situación más vulnerable, con menos probabilidades de realizar un cambio ascendente. Así mismo, la autora apunta de nuevo a la importancia de la situación familiar y del tipo de flujo migratorio para entender las estrategias de las mujeres inmigradas. En los casos en que las mujeres inmigran solas o son las pioneras de la migración familiar, la estrategia de trabajar como internas en el servicio doméstico les permite ahorrar el máximo de dinero posible en la fase inicial después de su llegada. A medida que el asentamiento en la nueva sociedad es mayor, o cuando el proceso de reagrupación se ha iniciado, la evolución normal es dejar el trabajo de internas por el de externas, y así poder vivir en su propio domicilio. I, con el transcurrir del tiempo, se abandona la idea de retorno y las mujeres inmigradas intentan abandonar el servicio doméstico para trabajar en otras actividades (residencias de ancianos, empresas de limpieza, hostelería) o crear su propio negocio. Este perfil difiere mucho de aquellas inmigradas que han llegado posteriormente a sus maridos, como es el caso mayoritario de las marroquíes, que se emplean en los hogares como externas por horas y compaginan el trabajo remunerado con el que realizan en sus familias.

Parella (2002) también afirma que la inserción laboral de la mujer inmigrante no puede ser analizada de manera estática. La autora argumenta que, aunque las opciones laborales de las mujeres inmigradas están, mayoritariamente, restringidas al servicio doméstico, y a pesar de las reducidas posibilidades laborales a las que pueden acceder, se producen trayectorias laborales diferenciadas según factores como el origen o la nacionalidad, la situación familiar, la magnitud del colectivo, la antigüedad del asentamiento o el nivel educativo.

En la misma línea, para el Colectivo Ioé (1998, 2001a y 2001b), esta aparente unidireccionalidad de las trabajadoras inmigradas hacia el servicio doméstico en los hogares españoles, no constituye necesariamente una condición irreversible, puesto que es posible el desplazamiento a otros sectores de actividad. Los autores reconocen que la posibilidad de pasar a trabajar en espacios públicos, fuera de la privacidad de los hogares, es de suma importancia, en cuanto el movimiento supone abandonar las prácticas de arbitrariedad y servidumbre que se atribuyen al servicio doméstico. Para los autores, esa probabilidad de ascenso laboral y de promoción social difiere en función del nivel educativo de la mujer inmigrante, del tiempo transcurrido desde su llegada a España, de su conocimiento del idioma o de su estructura familiar, entre otras variables. Para estos autores, cuando no se produce la movilidad laboral, las inmigradas se cronificarían en aquellos trabajos primeros a los que acceden, con lo cual se pueden generar nichos laborales cerrados, que fácilmente pueden desencadenar una segmentación étnica de la fuerza de trabajo. Esta situación, 
no obstante, también puede favorecer la creación de economías étnicas que permitan el surgimiento de empresarias prósperas, gracias a las redes de reciprocidad y a los vínculos culturales dentro de las comunidades de inmigradas.

En uno de los estudios del Colectivo Ioé (2003), se realiza una encuesta propia a partir de la cual se estudia la movilidad ocupacional de las extranjeras. Los principales resultados de esta investigación fueron que se da un punto de partida excesivamente dependiente del servicio doméstico, del que resulta difícil dar el salto hacia otras ocupaciones. Así, la mayoría de las entrevistadas que han logrado ocuparse en otras actividades, han estado en algún momento desde su llegada trabajando en el servicio doméstico. Los resultados apuntan que la movilidad ocupacional de las mujeres inmigradas no sólo se da del servicio doméstico interno al externo, sino que también es posible hacia otras actividades. De producirse, se dirige lentamente primero hacia otras ocupaciones poco prestigiadas (limpieza y hostelería) para luego ocuparse en las de mayor categoría laboral (oficinas y cuenta propia). Uno de los factores determinantes de la movilidad ascendente hacía otras actividades económicas que se observa en la investigación es la antigüedad de la residencia en España, aunque los autores apuntan que, para algunos colectivos, el tiempo desde la llegada parece no tener influencia alguna: las filipinas en el servicio doméstico y las chinas en la hostelería no presentan mayores probabilidades de abandonar estas ocupaciones en paralelo al tiempo de residencia en el país. No obstante, la conclusión genérica de la encuesta es que se produce una tendencia lenta a la movilidad ocupacional ascendente, aunque esta no se cumpla en todos los casos.

Los últimos estudios aparecidos sobre la movilidad laboral de las trabajadoras inmigrantes se han realizado a partir del análisis de los datos de la Encuesta Nacional de Inmigrantes del 2007 (ENI-2007), realizada por el Instituto Nacional de Estadística (ENI). La naturaleza retrospectiva de algunas de sus preguntas sobre la trayectoria laboral de las entrevistadas permite estudiar las características laborales previas a la migración, las del primer trabajo en España y las del momento de la entrevista, en 2007. Los resultados del análisis de Miret y Vidal (2009) confirman un descenso generalizado en la escala profesional en el primer trabajo en España, comparado con el que esas mujeres tenían en sus países de origen. De ese modo, la mayoría de las trabajadoras inmigradas se insertan en el segmento secundario: en el servicio doméstico especialmente, pero también en la hostelería, los servicios personales, el comercio y otros trabajos no cualificados. Por su lado, cuando se analiza la movilidad ocupacional de esas mujeres a lo largo de su estancia en España, el análisis demuestra que ésta es muy modesta y, cuando ocurre, se limita dentro del segmento secundario. Asimismo, la movilidad ascendente es más probable para aquellas mujeres con residencias más prolongadas en el país. Vono (2010) usa la ENI para estudiar el caso particular del colectivo latinoamericano y deduce el mismo resultado de ella: la mayor parte de estas mujeres encuentra su primer trabajo en el servicio doméstico (un 50\%) y su movilidad laboral posterior es muy reducida, puesto que se restringe al segmento secundario de la economía. 


\section{Referencias bibliográficas}

Anthias, F. (2000). «Metaphors of Home: Gendering New Migrations to Southern Europe». En: Anthias, F. y Lazaridis, G. (eds.). Gender and Migration in Southern Europe. Oxford: Berg, 15-47.

Arango, J. (2004). «La población inmigrada en España». Economistas, 99, 28-37.

CACHón, L. (1997). «Segregación sectorial de los inmigrantes en el mercado de trabajo en España». Cuadernos de Relaciones Laborales, 10, 49-73.

Carmona, S. (2000). «Inmigración y prostitución: el caso del Raval (Barcelona)». Papers: Revista de Sociologia, 60, 343-354.

Catarino, C. y Oso, L. (2000). «La inmigración femenina en Madrid y Lisboa: hacia una etnización del servicio doméstico y de las empresas de limpieza». Papers: Revista de Sociologia, 60, 183-207.

Colectivo Ioé (1998). «Inmigración y trabajo: hacia un modelo de análisis. Aplicación al caso de la construcción». Migraciones, 4, 35-70.

- (2001a). «Mujeres extranjeras en el mercado de trabajo español». Arxius, 5, 41-68.

- (2001b). Mujer, inmigración y trabajo. Madrid: Imserso. Colección Inmigración y Refugio.

- (2003). "Mujeres inmigradas y trabajo». En: Checa, F. (ed.). Mujeres en el camino: El fenómeno de la migración femenina en España. Barcelona: Icaria, 15-54.

Domingo, A. (1998). "La mujer inmigrada tras el velo estadístico». Papers de Demografia, 146, 1-16.

Domingo, A. y Brancós, I. (1999). «La población femenina de nacionalidad extranjera en la provincia de Barcelona, 1996». Papers: Revista de Sociologia, 60, 305-326.

Domingo, A. et al. (2007). «La población femenina extracomunitaria en España: Análisis territorial de los factores de inserción laboral». V Congreso sobre la Inmigración en España. Migraciones y Desarrollo Humano. Valencia: Universitat de València.

Fernández Cordón, J.A. (2004). «Demografía e inmigración». Economistas, 99, $16-27$.

GASPARD, F. (2000). «Invisibles, demonizadas, instrumentalizadas: las figuras de las inmigrantes y sus hijas». En: MARUANI, M. et al. (eds.). Las nuevas fronteras de la desigualdad. Barcelona: Icaria, 269-284.

IzQuierdo, A. (2000). «El proyecto migratorio de los indocumentados según género». Papers: Revista de Sociologia, 60, 225-240.

Juliano, D. (2000). «Mujeres estructuralmente viajeras: estereotipos y estrategias». Papers: Revista de Sociologia, 60, 381-389.

King, R. y Zontini, E. (2000). "The Role of Gender in the South European Immigration Model». Papers: Revista de Sociologia, 60, 35-52.

Kofman, E. (1999). "Female "Birds of Passage" a Decade Later: Gender and Immigration in the European Union». International Migration Review, 33 (2), 269-299.

Martínez Veiga, U. (1997). La integración social de los inmigrantes en España. Madrid: Trotta.

Miret, P. y Vidal, E. (2009). «Labour trajectories of immigrant women in Spain: towards a social upward mobility?». Papers de Demografia, 352, 1-30.

Oso, L. (1998). La migración hacia España de las mujeres jefas de hogar. Madrid: Ministerio de Trabajo y Asuntos Sociales. Instituto de la Mujer. Estudios, 52.

- (2003). "Las jefas de hogar en un contexto migratorio. Modelos y rupturas». En: Checa, F. (ed.). Mujeres en el camino: El fenómeno de la migración femenina en España. Barcelona: Icaria, 85-104. 
PARella, S. (2002). La internacionalización de la reproducción: La inserción laboral de la mujer inmigrante en los servicios de proximidad. Bellaterra: Universitat Autònoma de Barcelona. Departament de Sociologia. Facultat de Ciències Polítiques i Sociologia. Tesis doctoral.

Parella, S. y SAmper, S. (2007). «Factores explicativos de los discursos y estrategias de conciliación del ámbito laboral y familiar de las mujeres inmigradas no comunitarias en España». Papers: Revista de Sociologia, 85, 157-175.

Piore, M. (1971). "The Dual Labor Market: Theory and Implications». En: Gordon, D.M. (ed.). Political Economy: An Urban Perspective. Massachusetts: Health and Company, Lexington Books, 90-94.

- (1975). «Notes for a Theory of Labor Market Stratification». En: Edwards, R. et al. (eds.). Labor Market Segmentation. Massachusetts: D.C. Health and Company, Lexington Books, 125-150.

- (1979a). Birds of passage: migrant labour and industrial societies. Cambridge: Cambridge University Press.

- (1979b). "Los trabajadores extranjeros». En: Piore, M. (ed.). Paro e inflación. Perspectivas institucionales y estructurales. Madrid: Alianza, 273-289.

Solé, C. (2001). "La mujer inmigrante en la era de la globalización». En: RadL, R. (ed.). La mujer en la nueva era de la globalización. Madrid: Centro de Investigaciones Sociológicas (CIS).

- (2003). Inmigración, mercado de trabajo y género. Documento de trabajo. Serie Sociología, S2003/01.

VIDAL, E. (2009). Activitat i complementarietat sociodemogràfica entre les dones immigrades i les no immigrades a Espanya. Bellaterra: Universitat Autònoma de Barcelona. Departament de Geografia. Facultat de Filosofia i Lletres. Tesis doctoral.

Vono, D. (2010). ¿Preferidos y favorecidos?: El proceso de asentamiento de la población latinoamericana en España. Bellaterra: Universitat Autònoma de Barcelona. Departament de Geografia. Facultat de Filosofia i Lletres. Tesis doctoral. 